\title{
Estudio de las variables pedagógicas en tareas de enseñanza del fútbol en función de la parte de sesión
}

\section{Estudy of the pedagogical variables in teaching tasks of football based on the session part}

\author{
María de Gracia Gamero-Portillo ${ }^{1,2 *}$, Juan Manuel García-Ceberino ${ }^{1,2}$, Sebastián Feu ${ }^{1,2}$ y Antonio Antúnez \\ ${ }^{1}$ Facultad de Educación, Universidad de Extremadura (España). \\ ${ }^{2}$ Grupo de optimización del entrenamiento y rendimiento deportivo (GOERD), Universidad de Extremadura (España).
}

Resumen: El objetivo de este estudio fue analizar las variables pedagógicas de las tareas diseńadas por los profesores de pre-servicio para la enseñanza del futbol escolar en función de las partes de la sesión. Se han codificado 307 tareas diseñadas por 6 profesores en formación a través del Sistema Integral para el Análisis de las Tareas de Entrenamiento (SIATE). Se analizaron las variables pedagógicas que clasifica este sistema: situación de juego, presencia de portero, fase de juego, tipo de contenido I y II, contenido especifico, medio de enseñanza, nivel de oposición, tipo de participación y Feedback. Se realizó un análisis descriptivo e inferencial mediante la prueba Chi-Cuadrado, V de Cramer y los Residuos Tipificados Corregidos. Los resultados muestran diferencias significativas $(p<.05)$ en el diseño de las tareas para cada parte de la sesión con respecto a las variables pedagógicas, excepto en la variable tipo de contenido II.

Palabras claves: Tarea; profesor de pre-servicio; variable pedagógica, fútbol, SIATE.
Abstract: The objective of this study was to analyze the pedagogical variables of the tasks designed by the pre-service teachers for the teaching of school soccer based on the parts of the session. 307 tasks designed by 6 teachers in training have been codified through the Integral System for the Analysis of the Training Tasks (SIATE). The pedagogical variables classified by this system were analyzed: game situation, goalkeeper presence, game phase, content type I and II, specific content, teaching medium, level of opposition, type of participation and Feedback. A descriptive and inferential analysis was carried out using the Chi-Square test, Cramer's V test and the Corrected Typified Waste. The results show significant differences $(p<.05)$ in the design of the tasks for each part of the session with respect to the pedagogical variables, except in the variable type of content II.

Key words: Homework; pre-service teacher; pedagogical variable, soccer, SIATE.

\section{Introducción}

La Educación Física (EF) tiene como finalidad el desarrollo integral del niño, potenciando, su capacidad de autonomía y su plena integración social (Fernández, Cecchini y Zagalaz, 2002). Dentro de la EF se encuentra el deporte como contenido curricular y como el resto de contenidos curriculares, requiere de una planificación mediante el desarrollo de unidades didácticas (González-Espinosa, Ibáñez, y Feu, 2017). Según Ibáñez, $(2007,2008)$ la planificación y control del entrenamiento son métodos que favorecen la optimización del proceso de entrenamiento, por ello se ha convertido en la actualidad en una de las líneas emergentes dentro de la pedagogía deportiva. A su vez, dentro de la planificación deportiva es importante conocer los diferentes modelos de enseñanza-aprendizaje (E-A) que un docente tiene a su disposición para llevar a cabo el desarrollo de sus unidades didácticas; el estudio de éstos modelos de E-A sobre el deporte en edad escolar se ha convertido en un aspecto importante, en el que los investigadores/as buscan conocer la eficacia de cada uno de los modelos de E-A, así como las diferencias

Dirección para correspondencia [Correspondence address]: María de Gracia Gamero Portillo. E-mail: mgamerob@alumnos.unex.es entre ellos (Fletcher y Casey, 2014; Mitchell, Oslin y Griffin, 2003; Tejada, 2012).

Durante mucho tiempo, los modelos de E-A en la EF se centraban en la mera reproducción de los gestos técnicos del deporte a través de tareas analíticas, el conocimiento de las reglas del juego y la aplicación de lo aprendido en partidos al final de las clases. Según (Bunker; Thorpe, 1982) este modelo provocaba que los alumnos obtuvieran un escaso conocimiento de la dinámica intrínseca del juego. De esta forma, el éxito en el fútbol estará marcado por el resultado de la ejecución correcta de los diferentes aspectos técnicos que conforman el deporte como el tiro, el pase, el regate, la conducción... ignorando por tanto los aspectos tácticos que intervienen en la acción (Garganta, 2002). Por ello, en el ámbito educativo surgen nuevas formas que, influenciados por las corrientes pedagógicas vigentes, han generado métodos de enseñanza alternativos (Cañadas e Ibáñez, 2010). En esta línea, (Devís, 2004) plantea una "reconstrucción del deporte" dentro del ámbito escolar que presente a nuestros alumnos experiencias reflexivas que, a su vez, les proporcionen criterios con los que enfrentarse a toda la problemática socioeconómica, cultural y medioambiental que rodea el mundo del deporte en la sociedad actual. 
En el proceso de planificación de los deportes de invasión destacan las tareas, estas se definen como las unidades más concretas de la planificación deportiva (Sáenz-López, 2009). Las tareas permiten conocer qué tipo de metodología propone el entrenador, los contenidos del entrenamiento, los medios empleados o la carga de cada tarea (Gamonales, Gómez-Carmona, León, García-Santos, Gamero, Muñoz-Jiménez, 2019).

En la actualidad, con la finalidad de seguir profundizando y mejorando en el proceso de enseñanza aprendizaje del deporte, se observa que son diversos los autores los que han centrado su atención en el estudio de las metodologías utilizadas por los profesores/entrenadores para el diseño de las tareas y cómo éstas repercuten en el proceso de enseñanza-aprendizaje de los alumnos. (Gamonales et al. 2019; Cañadas, Ibañez, Feu, García y Parejo, 2011; González-Espinosa, Feu, GarcíaRubio, Antúnez, y García-Santos, 2017; Carrasco, Chirosa, Martín, Cajas, y Reigal, 2015; Gamero, García-Ceberino, González-Espinosa, Reina y Antúnez, 2017; García-Ceberino, Gamero, González-Espinosa, García- Rubio y Feu, 2018).

Por todo ello, el objetivo de este estudio fue analizar, las tareas de entrenamiento diseñadas por profesores en formación, atendiendo al uso que éstos hacen de las diferentes variables pedagógicas en función de las partes de sesión utilizadas en la planificación de unidades didácticas sobre el deporte de invasión en la edad escolar, en este caso, a través del fútbol. Su estudio ofrecerá un mayor conocimiento al contexto educativo sobre la planificación deportiva, favoreciendo la planificación de programas adecuados para el aprendizaje, pudiendo llegar a integrar la formación del jugador/aprendiz.

\section{Método}

\section{Diseño}

El diseño de la investigación se encuentra dentro de una estrategia asociativa de tipo comparativo y transversal, puesto que se persigue explorar la relación funcional existente entre variables en un momento determinado (Ato, López, y Benavente, 2013).

\section{Participantes y Muestra}

Los participantes de este estudio fueron seis profesores en fase de pre-servicio pertenecientes al grado de Educación Física de la Universidad de Extremadura, los cuales diseñaron para una de sus asignaturas de grado una unidad didáctica sobre el deporte de invasión el fútbol, las unidades didácticas debían estar formadas por un total de 12 sesiones. Por lo tanto, la muestra del estudio quedó formada por las tareas de enseñanza-aprendizaje que forman parte de las seis unidades didácticas planificadas por los profesores de pre-servicio, finalmente se analizaron un total de 307 tareas.

\section{Variables}

La variable independiente del estudio es las Partes de Sesión $(P S)$, dicha variable hace referencia a la estructura establecida en las sesiones de EF: animación, parte fundamental, culminación y vuelta a la calma (Sáenz-López, 1997). La parte de vuelta a la calma fue suprimida al utilizarse tan solo el $1 \%$ en las Unidades didácticas.

Las variables dependientes del estudio fueron las variables pedagógicas que definen una tarea. Dichas variables también se utilizan en el SIATE: situación de juego $(S J)$, presencia de portero $(P P)$, fase de juego $(F J)$, medio de enseńanza $(M E)$, tipo de contenido I (TCI), tipo de contenido II (TCII), contenido específico $(C E)$, nivel de oposición $(N O)$, tipo de participación $(T P)$ y, Feedback $(F B)$.

\section{Instrumento}

El instrumento empleado para la codificación de las tareas de futbol fue el SIATE (Ibáñez, Feu y Cañadas, 2016). El SIATE es un sistema metodológico utilizado para registrar y analizar los diferentes elementos que intervienen en el proceso de enseñanza de los deportes de invasión; es modulable debido a que se pueden definir la cantidad de información que los entrenadores registren de cada tarea de entrenamiento, por tanto, cuanta mayor información se consiga obtener de cada tarea de entrenamiento más profundo podrá ser el análisis y la evaluación del mismo. En esta ocasión, fueron registradas las variables pedagógicas que clasifica este sistema.

\section{Procedimiento}

En primer lugar, cada profesor de pre-servicio elaboró una unidad didáctica sobre el deporte de invasión del futbol. Una vez elaboradas y recopiladas las seis unidades didácticas se categorizaron mediante el instrumento SIATE, registrándose 307 tareas. Las tareas de las unidades didácticas fueron codificadas por un evaluador externo experto en la materia objeto de estudio, seguidamente se procedió al análisis de datos.

\section{Análisis estadístico}

En primer lugar, se realizó un análisis descriptivo para conocer las características de la muestra. Posteriormente, se llevó a cabo un análisis inferencial a través de la prueba $C h i-$ Cuadrado $\left(x^{2}\right)$, el coeficiente $V$ de Cramer $(V c)$, y se empleó los Residuos Tipificados Corregidos (RTC) de las tablas de contingencia para interpretar la relación y el grado de asociación entre la PS con el resto de variables pedagógicas estudiadas (Field, 2009). Además, se completó el análisis con la prueba exacta de Fisher debido a que en este estudio existen conteos muy bajos en diversas categorías de las variables pedagógicas. 


\section{Resultados}

En primer lugar, se ha analizado la frecuencia con la que los maestros de pre-servicio utilizan en sus unidades cada una de las partes de la sesión. Se observa en la Figura 1 que la parte de la sesión a la que más tareas dedican los profesores de pre-servicio durante el desarrollo de su unidad didáctica es la parte fundamental, le sigue la parte de animación y de culminación con un $24 \%$ y $23 \%$, utilizándose muy poco las tareas que pertenecen a la parte de vuelta a la calma, por ello, no vamos a profundizar en ella en el análisis de los resultados descriptivos y de los RTC.

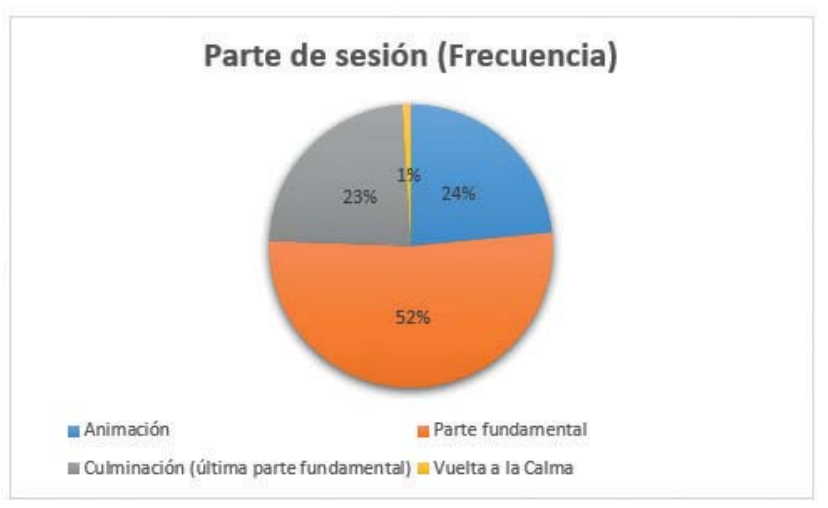

Figura 1. Gráfico sobre la frecuencia de las partes de la sesión en las U.D.

En las tablas 1, 2, 3 y 4 se muestran los resultados descriptivos y los $R T C$ de las variables pedagógicas que conforman el estudio para cada parte de sesión.

Tabla 1. Análisis descriptivo y los RTC de la variable pedagógica situación de juego para cada parte de sesión.

\begin{tabular}{|c|c|c|c|c|c|c|c|c|c|c|}
\hline \multirow[b]{2}{*}{ Variables } & \multirow[b]{2}{*}{ Categorías } & \multicolumn{3}{|c|}{ Animación } & \multicolumn{3}{|c|}{ Parte fundamental } & \multicolumn{3}{|c|}{ Culminación } \\
\hline & & $n$ & $\%$ & $R T C$ & $n$ & $\%$ & $R T C$ & $n$ & $\%$ & $R T C$ \\
\hline \multirow[t]{14}{*}{ Situación de juego } & 1vs0 & 12 & 16,7 & 0 & 28 & 17,5 & 4 & 8 & 11,1 & $-1,4$ \\
\hline & $1 \mathrm{vs} 1$ & 8 & 11,1 &,- 6 & 22 & 13,8 &, 4 & 10 & 13,9 &, 2 \\
\hline & $1 \mathrm{vs} 2$ & 2 & 2,8 &,- 3 & 6 & 3,8 & ,5 & 2 & 2,8 &,- 3 \\
\hline & $2 \mathrm{vs} 0$ & 9 & 12,5 & $-1,1$ & 37 & 23,1 & $3,2^{*}$ & 5 & 6,9 & $-2,5^{*}$ \\
\hline & $2 \mathrm{vs} 1$ & 0 & 0,0 & $-1,7$ & 6 & 3,8 & ,9 & 3 & 4,2 & ,7 \\
\hline & $3 \mathrm{vs} 0$ & 0 & 0,0 & $-1,2$ & 4 & 2,5 & 1,3 & 1 & 1,4 &,- 2 \\
\hline & $3 \mathrm{vs} 3$ & 1 & 1,4 &,- 6 & 2 & 1,3 & $-1,3$ & 4 & 5,6 & $2,1^{*}$ \\
\hline & $4 \mathrm{vs} 0$ & 2 & 2,8 &, 1 & 6 & 3,8 & 1,3 & 0 & 0,0 & $-1,6$ \\
\hline & $4 \mathrm{vs} 4$ & 1 & 1,4 &,- 2 & 4 & 2,5 & 1,3 & 0 & 0,0 & $-1,2$ \\
\hline & $5 \mathrm{vs} 5$ & 0 & 0,0 & $-2,0^{*}$ & 4 & 2,5 & $-1,6$ & 9 & 12,5 & $4,0^{*}$ \\
\hline & 6vs6 & 2 & 2,8 & ,1 & 3 & 1,9 &,- 8 & 3 & 4,2 & 1,0 \\
\hline & NvsN & 6 & 8,3 & $-1,1$ & 19 & 11,9 &,- 1 & 12 & 16,7 & 1,4 \\
\hline & Combinación & 6 & 8,3 & $4,5^{*}$ & 0 & 0,0 & $-2,6^{*}$ & 0 & 0,0 & $-1,4$ \\
\hline & $1 \mathrm{vs} \mathrm{N}$ & 11 & 15,3 & $4,1^{*}$ & 1 & 0,6 & $-3,4^{*}$ & 5 & 6,9 & ,6 \\
\hline
\end{tabular}

${ }^{*} R T C>|1,96|$ 
Tabla 2. Análisis descriptivo y los RTC de la variable pedagógica medio de enseñanza y fase de juego para cada parte de sesión.

\begin{tabular}{|c|c|c|c|c|c|c|c|c|c|c|}
\hline \multirow[b]{2}{*}{ Variables } & \multirow[b]{2}{*}{ Categorías } & \multicolumn{3}{|c|}{ Animación } & \multicolumn{3}{|c|}{ Parte fundamental } & \multicolumn{3}{|c|}{ Culminación } \\
\hline & & $n$ & $\%$ & $R T C$ & $n$ & $\%$ & $R T C$ & $n$ & $\%$ & $R T C$ \\
\hline \multirow[t]{2}{*}{ Presencia de portero } & Con portero & 2 & 2,8 & $-3,5$ & 24 & 15,0 &,- 6 & 24 & 33,3 & $4,5^{*}$ \\
\hline & Sin portero & 70 & 97,2 & $3,5^{*}$ & 136 & 85,0 & 6 & 48 & 66,7 & $-4,5^{*}$ \\
\hline \multirow[t]{9}{*}{ Medio de Enseñanza } & EAS & 26 & 36,1 &,- 4 & 69 & 43,1 & $1,9^{*}$ & 19 & 26,4 & $-2,3^{*}$ \\
\hline & EAC & 0 & 0,0 & $-1,9$ & 10 & 6,3 & $2,6^{*}$ & 1 & 1,1 & $-1,1$ \\
\hline & JSI & 22 & 30,6 & $4,1^{*}$ & 16 & 10,0 & $-2,7^{*}$ & 9 & 12,5 &,- 8 \\
\hline & JSE & 3 & 4,2 & 1,2 & 3 & 1,9 &,- 5 & 1 & 1,4 &,- 6 \\
\hline & JCI & 3 & 4,2 & 1,2 & 3 & 1,9 &,- 5 & 1 & 1,4 &,- 6 \\
\hline & JCE & 16 & 22,2 & 1,7 & 21 & 13,1 & $-1,4$ & 12 & 16,7 & ,2 \\
\hline & Predeporte & 0 & 0,0 & $-3,0^{*}$ & 13 & 8,1 &,- 4 & 14 & 19,5 & $3,6^{*}$ \\
\hline & Deporte & 0 & 0,0 & $-1,7$ & 3 & 1,9 & $-1,1$ & 6 & 8,3 & $3,1^{*}$ \\
\hline & Competición & 0 & 0,0 &,- 8 & 0 & 0,0 & $-1,5$ & 2 & 2,8 & $2,6^{*}$ \\
\hline \multirow[t]{4}{*}{ Fase de juego } & Ataque & 34 & 47,2 & $-1,8$ & 107 & 66,9 & $3,9^{*}$ & 32 & 44,4 & $-2,3^{*}$ \\
\hline & Defensa & 2 & 2,8 & ,6 & 3 & 1,9 &,- 1 & 1 & 1,4 &,- 4 \\
\hline & Mixto & 21 & 29,3 & $-1,3$ & 50 & 31,3 & $-1,7$ & 39 & 54,2 & $3,7^{*}$ \\
\hline & Calentamiento & 15 & 20,8 & $7,2^{*}$ & 0 & 0,0 & $-4,1^{*}$ & 0 & 0,0 & $-2,2^{*}$ \\
\hline
\end{tabular}

${ }^{*} R T C>|1,96|$

Nota: $E A S=$ Ejercicio de aplicación simple; EAC=Ejercicio de aplicación complejo; JSI = Juego simple inespecifico; JSE=Juego simple especifico; JCI=Juego complejo inespecifico; JCE=Juego complejo específico.

Tabla 3. Análisis descriptivo y los RTC de la variable pedagógica tipo de contenido, tipo de contenido II y contenido especifico para cada parte de sesión.

\begin{tabular}{|c|c|c|c|c|c|c|c|c|c|c|}
\hline \multirow[b]{2}{*}{ Variables } & \multirow[b]{2}{*}{ Categorías } & \multicolumn{3}{|c|}{ Animación } & \multicolumn{3}{|c|}{ Parte fundamental } & \multicolumn{3}{|c|}{ Culminación } \\
\hline & & $n$ & $\%$ & $R T C$ & $n$ & $\%$ & $R T C$ & $n$ & $\%$ & $R T C$ \\
\hline \multirow[t]{6}{*}{ Tipo de Contenido } & CTTIA & 6 & 8,3 & $-1,2$ & 21 & 13,1 & 4 & 11 & 15,3 & 9 \\
\hline & GTTIA & 20 & 27,8 &,- 5 & 54 & 33,8 & 1,5 & 18 & 25,0 & $-1,1$ \\
\hline & CTTGA & 13 & 18,1 &,-5 & 29 & 18,1 &,- 9 & 20 & 27,8 & 1,8 \\
\hline & GTTGA & 14 & 19,4 &,- 8 & 44 & 27,5 & $1,9^{*}$ & 13 & 18,1 & $-1,2$ \\
\hline & CTTEA & 1 & 1,4 & $-1,8$ & 8 & 5,0 &,- 4 & 8 & 11,1 & $2,4^{*}$ \\
\hline & Activación & 15 & 20,8 & $7,2^{*}$ & 0 & 0,0 & $-4,1^{*}$ & 0 & 0,0 & $-2,2^{*}$ \\
\hline \multirow[t]{5}{*}{ Tipo de contenido $\mathrm{II}^{1}$} & CTTID & 8 & 38,1 &, 2 & 24 & 48,0 & $2,3^{*}$ & 8 & 20,5 & $-2,6^{*}$ \\
\hline & GTTID & 1 & 4,8 & ,6 & 1 & 2,0 &,- 4 & 1 & 2,6 &,- 1 \\
\hline & CTTGD & 12 & 57,1 & 9 & 21 & 42,0 & $-1,2$ & 20 & 51,3 &, 5 \\
\hline & GTTGD & 0 & 0,0 &,- 7 & 0 & 0,0 & $-1,3$ & 2 & 5,1 & $1,9^{*}$ \\
\hline & CTTED & 0 & 0,0 & $-1,8$ & 4 & 8,0 &,- 9 & 8 & 20,5 & $2,4^{*}$ \\
\hline \multirow[t]{6}{*}{ Contenido Específico ${ }^{2}$} & Activación & 14 & 19,4 & $6,9^{*}$ & 0 & 0,0 & $-4,0^{*}$ & 0 & 0,0 & $-2,1^{*}$ \\
\hline & Conducción & 7 & 9,7 &,- 7 & 24 & 15,0 & 1,7 & 6 & 8,3 & $-1,1$ \\
\hline & Domino de balón & 4 & 5,6 & 1,3 & 4 & 2,5 &,- 8 & 2 & 2,8 &,- 3 \\
\hline & Contenidos Ataq-Def. & 0 & 0,0 & $-3,0^{*}$ & 10 & 6,3 & $-1,6$ & 17 & 23,6 & 5,1 \\
\hline & Tiro & 5 & 6,9 &,- 2 & 13 & 8,1 &, 4 & 5 & 6,9 &,- 2 \\
\hline & Pase-Control & 8 & 11,1 &,- 8 & 28 & 17,5 & 1,8 & 7 & 9,7 & $-1,2$ \\
\hline
\end{tabular}

${ }^{*} R T C>|1,96|$

Nota: CTTIA=Conducta táctico-técnica individual de ataque; CTTID=Conducta táctico-técnica individual de defensa; GTTIA=Gesto técnico-táctico individual de ataque $G T T I D=G e s t o$ técnico-táctico individual de defensa; CTTGA=Conducta táctico-técnica grupal de ataque; CTTGD=Conducta táctico-técnica grupal de defensa; GTTGA=Gestos técnico-tácticos grupales de ataque; GTTGD=Gestos técnico-tácticos grupales de defensa; CTTEA=Conducta táctico-técnica de equipo de ataque; CTTED=Conducta táctico-técnica de equipo de defensa; GTTEA = Gestos técnico-tácticos de equipo de ataque; GTTED= Gestos técnico-tácticos de equipo de defensa.

${ }^{1}$ Para las tareas de enseñanza que trabajan una fase de juego mixta.

${ }^{2}$ En la variable contenidos específicos se han resumido los resultados. 
Tabla 4. Análisis descriptivo y los $R T C$ de la variable pedagógica nivel de oposición, tipo de participación y feedback para cada parte de sesión.

\begin{tabular}{|c|c|c|c|c|c|c|c|c|c|c|}
\hline \multirow[b]{2}{*}{ Variables } & \multirow[b]{2}{*}{ Categorias } & \multicolumn{3}{|c|}{ Animación } & \multicolumn{3}{|c|}{ Parte fundamental } & \multicolumn{3}{|c|}{ Culminación } \\
\hline & & $n$ & $\%$ & $R T C$ & $n$ & $\%$ & $R T C$ & $n$ & $\%$ & $R T C$ \\
\hline \multirow[t]{5}{*}{ Nivel de Oposición } & Sin oposición & 30 & 41,7 &, 4 & 70 & 43,8 & 1,6 & 18 & 25,0 & $5,9^{*}$ \\
\hline & Obstáculos estáticos & 3 & 4,2 & $-1,8$ & 22 & 13,8 & $2,7^{*}$ & 4 & 5,6 & $-1,3$ \\
\hline & Obstáculos dinámicos & 0 & 0,0 &,- 6 & 0 & 0,0 & $-1,0$ & 1 & 1,4 & 1,8 \\
\hline & Oposición modulada & 0 & 0,0 &,- 8 & 2 & 1,3 & 1,4 & 0 & 0,0 &,- 8 \\
\hline & Oposición & 39 & 54,2 & ,8 & 66 & 41,3 & $-3,3^{*}$ & 49 & 68,1 & $3,5^{*}$ \\
\hline \multirow[t]{3}{*}{ Tipo de participación } & Simultánea & 64 & 88,9 & $4,0^{*}$ & 103 & 64,4 & $-2,3^{*}$ & 45 & 62,5 & $-1,6$ \\
\hline & Alternativa & 0 & 0,0 & $-2,6^{*}$ & 9 & 5,6 &,- 9 & 12 & 16,7 & $3,8^{*}$ \\
\hline & Consecutiva & 8 & 11,1 & $-2,8^{*}$ & 48 & 30,0 & $3,0^{*}$ & 15 & 20,8 &,- 5 \\
\hline \multirow[t]{3}{*}{ Feedback } & Motivacional & 21 & 29,2 & $6,8^{*}$ & 3 & 1,9 & $-4,6^{*}$ & 4 & 5,6 & $-1,2$ \\
\hline & Descriptivo/Prescriptivo & 29 & 40,3 & $-2,1^{*}$ & 97 & 60,6 & $3,5^{*}$ & 31 & 43,1 & $-1,6$ \\
\hline & Interrogativo & 22 & 30,6 & $-1,6$ & 60 & 37,5 &,- 5 & 37 & 51,4 & $2,5^{*}$ \\
\hline
\end{tabular}

${ }^{*} R T C>|1,96|$

El análisis descriptivo de los datos muestra cuales son las categorías más utilizadas en cada una de las variables pedagógicas para cada parte de sesión.

Los profesores en fase de pre-servicio, en la parte de animación, tienden a diseñar ejercicios de aplicación simple $(36,1 \%)$ y juegos simples inespecificos (30,6\%), en fase de juego de ataque (47,2\%), sin presencia de portero (97,2\%), con trabajo de gestos técnico-tácticos individuales de ataque (27,8\%) y con contenidos de activación (20,8\%), en situaciones de juego $\operatorname{lvs} 0(16,7 \%)$ y $1 v s N(15,3 \%)$. Entre los contenidos específicos destaca los contenidos de activación (19,4\%). Así mismo, predominan las tareas con oposición $(54,2 \%)$, con participación simultánea $(80,9 \%)$ y con un feedback descriptivo (40,3\%).

En la parte fundamental, tienden a diseñar ejercicio de aplicación simple (43,1\%), en fase de juego de ataque (66,9\%), sin presencia de portero $(85,0 \%)$, con trabajo de gestos técnicostácticos individuales de ataque (33,8\%) y gestos técnicos-tácticos grupal de ataque (27,5\%) en situaciones de juego $2 v s 0(23,1 \%)$ y $1 v s 0(17,5 \%)$. Entre los contenidos específicos destaca el $p a-$ se-control (17,5\%) y la conducción (15,0\%). En cuanto al tipo de contenidos II destaca los contenidos táctico-técnicos individuales de defensa (48\%) y los contenidos tácticos-técnicos grupales de defensa (42,0\%). Así mismo, predominan las tareas sin oposición (43,8\%), con participación simultánea $(64,4 \%)$ y con un feedback descriptivo $(60,6 \%)$.

En la parte de culminación, tienden a diseñar ejercicios de aplicación simple (26,4\%) y tareas de pre-deporte (19,5\%), en fase de juego mixta $(54,2 \%)$ y de ataque $(44,4 \%)$, sin presencia de portero $(66,7 \%)$, con trabajo de conductas tácticotécnicas grupales de ataque $(27,8 \%)$ y conductas táctico-técnicos grupales de defensa (51,3\%), predominan las situaciones de juego $N v s N(16,7 \%)$ y Ivsl (13,9\%). Entre los contenidos específicos destacan los contenidos de ataque-defensa (23,6\%).
Así mismo, predominan las tareas con oposición (68,1\%), con participación simultánea $(62,4 \%)$ y con un feedback interrogativo (51,4\%).

Los RTC de las tablas de contingencia ofrecen información sobre la asociación entre de las categorías de codificación de las variables pedagógicas y las partes de sesión. Durante la parte de animación, los $R T C$ indican más casos de los esperados en: la situación de juego $1 \mathrm{vsN}(R T C=4,1)$ y las situaciones combinadas $(R T C=4,5)$, la fase de Calentamiento $(R T C=7,2)$, el tipo de contenido de activación $(R T C=7,2)$, el juego simple inespecífico $(R T C=4,1)$, las tareas sin presencia de portero $(R T C=3,5)$, con participación simultánea $(R T C=4,0)$ y con un feedback motivacional $(R T C=6,8)$. El contenido específico con más casos de los esperados es el contenido de activación $(R T C=6,9)$.

En la parte fundamental, los $R T C$ señalan más casos de los esperados en: el ejercicio de aplicación simple y complejo $(R T C=1,9$ y 2,6$)$, respectivamente, las situaciones de juego $2 \mathrm{vs} 0(R T C=3,2)$, la fase de juego de ataque $(R T C=3,9)$, en cuanto al tipo de contenido I y II destacan las tareas con gestos técnicos-tácticos grupales de ataque $(R T C=1,9)$ y las conductas tácticos-técnicas individuales de defensa $(R T C=2,3)$, las tareas con obstáculos estáticos $(R T C=2,7)$, con participación consecutiva $(R T C=3,0)$ y con un feedback prescriptivo $(R T C=3,5)$.

Finalmente, durante la parte culminación, los $R T C$ indican más casos de los esperados en: las situaciones de juego $3 \times 3$ y $5 \times 5(R T C=2,1$ y 4,0), respectivamente, la fase de juego mixta $(R T C=3,7)$, las tareas con presencia de portero $(R T C=4,5)$ las conductas táctico-técnicas de equipo en ataque $(R T C=2,4)$, como contenido específico los contenidos de ataque-defensa $(R T C=5,1)$, el pre-deporte $(R T C=3,6)$, deporte $(R T C=3,1)$ y la competición $(R T C=2,6)$ como medio de en- 
señanza. Las tareas sin oposición $(R T C=5,9)$ y con oposición $(R T C=3,5)$ y con una participación alternativa $(R T C=3,8)$ y un feedback interrogativo $(R T C=2,5)$.
Los resultados inferenciales de las variables pedagógicas con la prueba Chi-Cuadrado y la prueba de Fisher se exponen en la Tabla 5.

Tabla 5. Resultados inferenciales con la prueba Chi-Cuadrado y grado de asociación en función de la parte de sesión.

\begin{tabular}{|c|c|c|c|c|c|c|c|}
\hline & $x^{2}$ & $g l$ & $p$ & $f$ & $p$ & $V c$ & $p$ \\
\hline Situación de juego & 148,746 & 96 &, $000^{*}$ & 171,870 &, $000^{*}$ &, 402 &, $000^{*}$ \\
\hline Presencia de portero & 25,761 & 3 &, $000^{*}$ & 25,607 &, $000^{*}$ &, 290 &, $000^{*}$ \\
\hline Fase de Juego & 370,761 & 12 &, $000^{*}$ & 81,763 &, $000^{*}$ &, 634 &, $000^{*}$ \\
\hline Tipo de contenido I & 383,950 & 36 &, $000^{*}$ & 105,387 &, $000^{*}$ &, 646 &, $000^{*}$ \\
\hline Tipo de contenido II & 15,435 & 8 &, 051 & 14,515 & $, 0,27$ &, 265 &, 051 \\
\hline Contenido Específico & 476,571 & 162 &, $000^{*}$ & 210,025 &, $007^{*}$ &, 719 &, $000^{*}$ \\
\hline Medio de enseńanza & 68,925 & 24 &, $000^{*}$ & 73,255 &, $000^{*}$ &, 274 &, $000^{*}$ \\
\hline Nivel de oposición & 28,110 & 12 &, $005^{*}$ & 30,473 &, $003^{*}$ &, 175 &, $005^{*}$ \\
\hline Tipo de participación ${ }^{1}$ & 29,614 & 6 &, $000^{*}$ & 28,446 & $.000^{*}$ & ,297 &, $000^{*}$ \\
\hline Feedback & 358,581 & 9 &, $000^{*}$ & 78,053 & $.000^{*}$ &, 624 &, $000^{*}$ \\
\hline
\end{tabular}

'para esta variable se utilizó el Cc en lugar de la $V c ;{ }^{*} p<, 05$

Los resultados inferenciales ponen de manifiesto la existencia de diferencias estadísticamente significativas $(p<, 05)$ en el diseño de las tareas para cada parte de sesión con respecto a las variables pedagógicas, excepto en la variable tipo de contenido II $(p=, 051)$. Los resultados obtenidos mediante la prueba exacta de Fisher coinciden con los resultados del ChiCuadrado.

El coeficiente de $V$ de Cramer señala una fuerza de asociación baja en las variables presencia de portero, tipo de contenido II, medio de enseñanza, nivel de oposición y tipo de participación $\left(V_{c \leq} \leq 299\right)$; una fuerza de asociación moderada ( $V c$ entre 300 y ,499) en la variable situación de juego $(V c=, 402)$; y una fuerza de asociación alta en las variables fase de juego, tipo de contenido I, contenido específico y feedback $\left(V_{c}>, 500\right)$.

\section{Discusión}

Conocer el diseño de las tareas de enseñanza mediante el uso que los profesores hacen de las variables pedagógicas resulta fundamental para la optimización del aprendizaje. Por ello, este estudio tuvo por objetivo analizar las variables pedagógicas empleadas por los profesores de pre-servicio durante el diseńo de las tareas para la enseńanza del futbol escolar en función de las partes de sesión en Educación Física: animación, parte fundamental y culminación. Los resultados ponen de manifiesto la existencia de diferencias estadísticamente significativas en el diseño de las tareas para cada parte de sesión con respecto a las variables pedagógicas excepto la variable tipo de contenido II.

Atendiendo a la asociación entre las categorías de cada variable, cabe decir que es alta en la variable de fase de juego, tipo de contenido I, contenido específico y feedback. En la parte de animación existen más casos de los esperados en aquellas tareas que trabajan los contenidos de calentamiento a través de juegos simples y de situaciones de $1 \mathrm{vsN}$. En la parte fundamental se hace más hincapié en las tareas con oposición de obstáculos estáticos, más individualizadas y se imparten a través de ejercicios simples y complejos. Sin embargo, en la parte final de la sesión (culminación), antes de la vuelta a la calma, existen más casos de los esperados en las situaciones reducidas y de igualdad numérica, que, además, se aproximan al deporte real, trabajándose los objetivos mediante el juego complejo específico, el pre-deporte y el deporte, autores como (Garganta, 1997; Sáenz-López, 2009; Ibáńez, 2000) afirman que el medio de entrenamiento que más se debería utilizar y fomentar en esta etapa formativa por su carácter lúdico, socializador y por respetar la estructura del juego real es el juego.

El uso que los profesores hacen de estas variables implica una progresión en cada una de sus sesiones. Para el desarrollo de los objetivos en la parte de animación y fundamental utilizan un método analítico, basado en la repetición de ejercicios y en la mejora de ciertas habilidades individuales (Méndez, 2005), pretendiendo llegar a la práctica global del juego desde la técnica individual aislada y descontextualizada (Blázquez, 1999), el predominio de ejercicios, juegos inespecíficos y situaciones sin oponente, indica que los maestros de pre-servicio dan predominio al aprendizaje técnico sobre táctico (Feu, García-Rubio, Gamero e Ibáńez, 2019). Sin embargo, en la parte final de la sesión (culminación) se observa un intento de combinar la metodología analítica con una metodología 
más globalizada que se asemeje a la práctica del juego real, mediante la interacción de compañeros y la modificación de diversos aspectos del juego, donde los alumnos alcanzan la práctica global del deporte desde la toma de decisión (Balakrishnan, Rengasamy y Aman, 2011). Un estudio realizado sobre estilos de enseñanzas aplicados por diferentes entrenadores, también señala al modelo tradicional como uno de los más utilizados entre la muestra objeto de estudio, la cual estuvo formada por un total de 26 entrenadores (Zurita, Pérez, González, Castro, Chacón y Ambris, 2016).

Los resultados de este estudio coinciden en gran medida con estudios similares dirigidos al análisis de las variables pedagógicas empleadas por los profesores en fase de pre-servicio para la enseñanza del balonmano en el contexto escolar. Gamero, Reina, Antúnez, y Feu (2018), en su estudio, encuentran diferencias significativas en el diseño de las tareas para cada parte de sesión en todas las variables pedagógicas, menos en las variables de contenido específico y feedback que no fueron objeto de estudio. A pesar de ello, en ambos estudios, los profesores, en la parte de animación, utilizan con más frecuencia la situación 1vs0, el EAS y la participación simultánea. En la parte fundamental, la situación 1vs0, el EAS, la fase de ataque, las tareas sin oposición y como contenido, los GTTIA. Por último, en la parte de culminación coinciden en la situación 1vs0, el EAS y la participación simultánea. La variable pedagógica que más definen una tarea es el medio de enseñanza, en ambos estudios emplean mayoritariamente el medio EAS en cada parte de la sesión, utilizando prácticamente la misma metodología analítica a lo largo de la clase; esta variable es la más importante dado que modifica el resto de variables en dónde existen diferencias significativas (González-Espinosa et al., 2017). El estudio realizado por (Bolotin y Bakayev, 2017) reveló que una de las condiciones más importante y que determina la efectividad de los jóvenes jugadores es la selección efectiva de los medios de entrenamiento por parte de los entrenadores.

\section{Conclusiones}

Los profesores de pre-servicio hacen diferente uso de las distintas variables pedagógicas en función de la parte de sesión en la que se encuentren, excepto en la variable tipo de contenido II debido a que esta variable solo se codifica cuando se trabaja la fase de juego mixta. Las diferencias más significativas en función de las partes de la sesión se encuentran en las variables: fase de juego, tipo de contenido I, contenido específico y feedback.

El uso que los profesores de pre-servicio hacen de las diferentes variables pedagógicas en cada parte de sesión, ubican a sus unidades didácticas en un modelo de enseñanza tradicional, aunque se aprecia una tendencia de combinar las tareas analíticas con las tareas globales, como ocurre en la parte de culminación.

Los datos de esta investigación permiten conocer la metodología utilizada por los profesores de pre-servicio en la planificación de sus unidades didácticas sobre el deporte del fútbol y el uso que éstos hacen de las diferentes variables pedagógicas, dando información sobre la forma en que entienden y plantean la enseńanza deportiva en el ámbito escolar.

\section{Bibliografía}

1. Ato, M., López, J. J., y Benavente, A. (2013). Un sistema de clasificación de los diseños de investigación en psicología. Anales de Psicología, 29(3), 1038-1059. doi:10.6018/analesps.29.3.178511

2. Balakrishnan, M., Rengasamy, S., y Aman, M.S. (2011). Effect of Teaching Games for Understanding Approach on Students' Cognitive Learning Outcome. International Scholarly and Scientific Research \& Innovation, 5(5), 714-716.

3. Blázquez, D. (1999). La iniciación deportiva y el deporte escolar. Barcelona: Inde.

4. Bolotin, A. y Bakayev, V. (2017). Pedagogical conditions necessary for effective speed-strength training of young football players (15-17 years old). Journal of Human Sport and Exercise, 12(2), 405-413. doi: https:// doi.org/10.14198/jhse.2017.122.17

5. Bunker, D. y Thorpe, R. (1982). A model for the teaching of games in secondary schools. Bulletin of Physical Education, London, 18(1), 5-8.

6. Cañadas, M., e Ibáńez, S. J. (2010). La planificación de los contenidos de entrenamiento de baloncesto en equipos de iniciación. E-balonmano. com: Revista de Ciencas del Deporte, 6(1), 49-65.

7. Cañadas, M., Ibáñez, S. J., Feu, S., García, J., y Parejo, I. (2011). Análisis de los medios de entrenamiento en un equipo minibasket y la influencia de un programa formativo para el entrenador. Un estudio de caso. Ágora para la Educación Física y el Deporte, 13(3), 363- 382.

8. Carrasco, H., Chirosa, L.J., Martín, I., Cajas, B., y Reigal, R.E. (2015).
Efectos de un programa extraescolar basado en juegos reducidos sobre la motivación y las necesidades psicológicas básicas en las clases de educación física. Revista Iberoamericana de Psicología del Ejercicio y el Deporte, $10(1), 23-31$.

9. Devís, J. (2004). El deporte en la escuela: posibilidades educativas para el nuevo milenio. En V. López Pastor, R. Monjas Aguado y A. Fraile Aranda (Coord.), Los últimos diez años de la Educación Física escolar (pp. 77-85). Valladolid. Universidad de Valladolid.

10. Field, A. (2009). Discovering statistics using SPSS (3a ed.). London: Sage Publications Ltd.

11. Fernández, E., Cecchini, J. A., y Zagalaz, M. L. (2002). Didáctica de la educación física en la educación primaria. Madrid, Síntesis.

12. Feu, S., García-Rubio, J., Gamero, M.G., e Ibáńez, S.J. (2019) Planificación de tareas para el aprendizaje deportivo por parte de profesores de educación física en la fase previa al servicio. PLoS ONE 14 (3): e0212833. https://doi.org/10.1371/journal.pone.0212833

13. Fletcher, T., y Casey, A. (2014). The challenges of models-based practice in physical education teacher education: A collaborative self-studyGamero, M. G., García-Ceberino, J. M., González-Espinosa, S., Reina, M., y Antúnez, A. (2017). Análisis de las variables pedagógicas en las tareas diseñadas para el balonmano en función del género de los docentes. E-Balonmano.com: Revista de Ciencias del Deporte, 13(3) 217-224.

14. Gamonales Puerto, J.M.; Gómez Carmona, C.D.; León Guzmán, 
K.; García Santos, D.; Gamero Portillo, M.G. y Muñoz Jiménez, J. (2019). Análisis de las tareas de entrenamiento en fútbol-base: diferencias entre dos meses durante el periodo competitivo en la categoría sub-19. SportisSci J, 5(1),30-52. DOI: https://doi.org/10.17979/sportis.2019.5.1.3469

15. García-Ceberino, J.M., Gamero, M. G., González-Espinosa, S., GarcíaRubio, J., y Feu, S. (2018). Estudio de la carga externa de las tareas para la enseñanza del balonmano en función del género de los profesores en formación. E-balonmano.com: Revista de Ciencias del Deporte 14(1), $45-54$.

16. Garganta, J. (1997). Para una teoría de los juegos deportivos colectivos. En A. Graça, y J. Oliveira (Eds.), La enseñanza de los juegos deportivos (pp. 9-24). Barcelona: Paidotribo.

17. Garganta, J. (2002). O treino da táctica e da técnica nos jogos desportivos á luz do compromisso cognição-acção. En V. J. Barbanti, A. C. Amadio, J. O. Bento \& A. T. Marques (2002), Esporte e Atividade Física (pp. 281-306). Brasil.

18. González-Espinosa, S.J., Ibáńez, S., y Feu, S. (2017). Diseño de dos programas de enseńanza del baloncesto basados en métodos de enseñanza-aprendizaje diferentes. E-Balonmano.com: Revista de Ciencias del Deporte, 13(2) 131-152.

19. González-Espinosa, S., Feu, S., García-Rubio, J., Antúnez, A., y GarcíaSantos, D. (2017). Diferencias en el aprendizaje según el método de enseñanza-aprendizaje en el baloncesto. Revista de Psicología del Deporte, 26(1), 65-70.

20. Ibáńez, S. J. (2000). La enseńanza del baloncesto dentro del contexto educativo. Habilidad motriz. Revista de Ciencias de la Actividad Física y del Deporte, 15, 12-21.

21. Ibáńez, S. J. (2007). El control del entrenamiento como medio de for- mación permanente del entrenador de baloncesto. En S. J. Ibáńez, S. Feu, I., Parejo, J. García y M. Cañadas. (Eds.), IV Congreso Ibérico de Baloncesto. Desde la base a la élite deportiva. Libro de resúmenes y programa oficial (pp. 109). Cáceres: Copegraf.

22. Ibáńez, S. J. (2008). La planificación y el control del entrenamiento técnico-táctico en baloncesto. En N. Terrados y J. Calleja (Coord.), Fisiología, entrenamiento y medicina del baloncesto (pp. 299-313). Barcelona: Paidotribo.

23. Ibáńez, S. J., Feu, S., y Cañadas, M. (2016). Sistema integral para el análisis de las tareas de entrenamiento, SIATE, en deportes de invasión. E-Balonmano.com: Revista de Ciencias del Deporte, 12(1), 3-30.

24. Méndez, A. (2005). Técnicas de enseñanza en la iniciación al baloncesto. Zaragoza: Inde.

25. Mitchell, S. A., Oslin, J. L., y Griffin, L. L. (2003). Sport foundations for elementary physical education: A tactical games approach: ERIC.

26. Sáenz-López, P. (1997). La Educación Física y su Didáctica. Manual para el profesor. Sevilla: Wanceulen.

27. Sáenz-López, P. (2009). Diseño de tareas tácticas y técnicas en la iniciación al baloncesto. En G. Ortega y A. C. Jiménez (Coord.), Táctica y Técnica en la Iniciación al Baloncesto (pp. 117-133). Sevilla: Wanceulen.

28. Tejada, C. P. (2012). Efecto del entrenamiento mediante el método comprensivo en ultimate frisbee. Revista Internacional de Medicina y Ciencias de la Actividad Física y del Deporte (46), 10-10.

29. Zurita Ortega, F., Pérez Cortés, A. J., González Campos, G., Castro Sánchez, M., Chacón Cuberos, R. y Ambris Sandoval, J. (2016). Estilos de enseñanza-entrenamiento y relación con ansiedad de jugadores de iniciación al futbol. Sportis. Scientific Journal of School Sport, Physical Education and Psychomotricity, 2(3), 390-411. Doi: https://doi. org/10.17979/sportis.2016.2.3.1721 